\title{
Stability and Deformation of Vesicles in Cylindrical
}

\author{
Flow
}

Dan Liu ${ }^{a}$, Zhihao Zhang ${ }^{a}$, Rong Wang ${ }^{a, *}$ and Jinglei Hu ${ }^{b, *}$

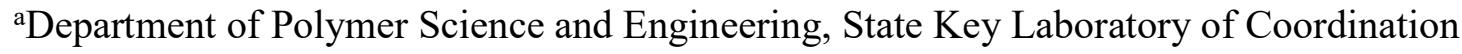
Chemistry and Collaborative Innovation Center of Chemistry for Life Sciences, Key Laboratory of High Performance Polymer Material and Technology of Ministry of Education, School of Chemistry and Chemical Engineering, Nanjing University, Nanjing 210023, China.

${ }^{\mathrm{b}}$ Kuang Yaming Honors School \& Institute for Brain Sciences, Nanjing University, Nanjing,210023, China.

*Email: wangrong@nju.edu.cn

*Email: hujinglei@nju.edu.cn 


\section{More Detailed Information about DPD Methods}

Dissipative particle dynamics (DPD) was proposed by Hoogerbrugge and Koelman in 1992 to model complex hydrodynamic phenomena. ${ }^{1}$ The motion of particles follows Newton's equation of motion. It satisfies ${ }^{2}$

$$
\begin{gathered}
m_{i} \frac{\mathrm{d} \vec{v}_{i}}{\mathrm{~d} t}=\vec{f} \\
\vec{f}=\vec{f}^{\mathrm{ex}}+\vec{f}^{\mathrm{in}}
\end{gathered}
$$

Among them, $m_{i}, v_{i}$, and $\vec{f}_{i}$ are the mass, velocity, and force of the $i$ th particle, respectively. We set the mass of all particles to $1 . \vec{f}_{i}^{\text {in }}$ includes three parts: conservative force. $\vec{f}_{i j}^{\mathrm{C}}$, dissipative force $\vec{f}_{i j}^{\mathrm{D}}$, and random force $\vec{f}_{i j}^{\mathrm{R}} \cdot \vec{f}_{i}^{\mathrm{ex}}$ is the external force exerted on the solvent particles.

$$
\vec{f}^{\mathrm{in}}=\sum_{j \neq i}\left(\vec{f}^{\mathrm{C}}+\vec{f}^{\mathrm{D}}+\vec{f}^{\mathrm{R}}\right)
$$

The internal force is the sum of all the other particles in a certain range, i.e. the cutoff radius $r_{\mathrm{c}}$. In this study, the cutoff radius is taken as the unit length, $r_{\mathrm{c}}=1$. Conservative force, random force, and dissipative force are all paired, which are given by the following formulae (S4)-(S6).

$$
\vec{f}^{\mathrm{C}}=a_{i j} \omega\left(r_{i j}\right) \hat{r}_{i j}
$$

Here $a_{i j}$ refers to the repulsive interaction parameter between particles $i$ and $j$, which reflects the chemical properties of the interacting particles. $\vec{r}_{i j}=\vec{r}_{i}-\vec{r}_{j}, \hat{r}_{i j}=\vec{r}_{i j} / r_{i j}, r_{i j}=\left|\vec{r}_{i j}\right| \omega\left(r_{i j}\right)$ is the weight function, providing a range of interaction for DPD particles : $\omega\left(r_{i j}\right)=1-r_{i j} / r_{c}\left(r_{i j} \leq\right.$ $\left.r_{c}\right) ; \omega\left(r_{i j}\right)=0\left(r_{i j}>r_{c}\right)$

$$
\vec{f}^{D}=-\gamma \omega^{2}\left(r_{i j}\right)\left(\hat{r}_{i j} \cdot \vec{v}_{i j}\right) \hat{r}_{i j}
$$

where friction coefficient $\gamma$ governs the magnitude of the dissipative force and $\vec{v}_{i j}=\vec{v}_{i}-\vec{v}_{j}$

$$
\vec{f}^{\mathrm{R}}=\sigma \omega\left(r_{i j}\right) \theta_{i j} \hat{r}_{i j}
$$


The parameter $\theta_{i j}$ is a randomly fluctuating variable with Gaussian statistics and $\sigma^{2}=2 \gamma k_{\mathrm{B}} T$.

We employ the finitely extensible nonlinear elastic (FENE) potential between the consecutive particles to link the connected beads of the diblock copolymer. ${ }^{3}$ The FENE potential is $V_{\mathrm{FENE}}\left(r_{i j}\right)=-0.5 k R_{0}^{2} \operatorname{In}\left[1-\left(r_{i j} / R_{0}\right)^{2}\right]$ when $r_{i j}<R_{0}$ and $V_{\mathrm{FENE}}\left(r_{i j}\right)=\infty$ when $r_{i j} \geq R_{0}$. We take the bond constant $k=50$ and $R_{0}=1.5 r_{\mathrm{c}}$. The polymer concentration is set to 0.1 . The time integration of Newton's equation of motion was completed by applying the modified velocityVerlet algorithm with $\lambda=0.65$ and then $\Delta t=0.03 .{ }^{2}$ When $f^{\mathrm{ex}}=24$ and $C_{\mathrm{d}}=0.8$, the $x$-component of the velocity profile of solvent particles with respect to the average velocity are drawn along the $r$ direction in the Figure S1. The amplitude of random noise is set as $\sigma=3.0$. The simulation of the initial vesicle was created in a cubic box of $25^{3}$ with a number density of 3 under periodic boundary conditions. The initial vesicles are designed to be studied as objects in a cylindrical channel system.

The results about the mechanical tension $\Sigma_{\text {mec }}$ refer to $\Sigma_{\text {mec }}=\int_{-\infty}^{+\infty} s(z) \mathrm{d} z \cong \int_{-L_{Z} / 2}^{+L_{Z} / 2} s(z) \mathrm{d} z$ in Figure S2 (b). In addition, the stress profile $s(z)=\sum_{\mathrm{T}}(z)-\sum_{\mathrm{N}}=-\left[P_{\mathrm{T}}(z)-P_{\mathrm{N}}\right]$ and the local pressure tensor $P_{\alpha \beta}(z)=\frac{1}{\Omega}\left\langle\sum_{i=1}^{\mathrm{N}} m_{i}\left(v_{i}\right)_{\alpha}\left(v_{i}\right)_{\beta} \Lambda_{\mathrm{i}}+\sum_{i=1}^{\mathrm{N}-1} \sum_{j>i}^{\mathrm{N}}\left(r_{i j}\right)_{\alpha}\left(f_{i j}\right)_{\beta} l_{i j}\right\rangle$ are obtained by building the bilayer with different $A$ (projected area per lipid) ${ }^{4}$, shown in Figure S2 (c). Figure S2 (d) is the relative density profile with $A=1.025$. The results of the average fluidic flow velocity $\bar{v}$ exhibit in Figure S3. The $A, l_{\mathrm{me}}, \Omega, l_{\mathrm{ij}}, \Lambda_{\mathrm{ij}}, P_{\mathrm{N}}, P_{\mathrm{T}}$ above are the projected area per lipid, the membrane thickness, volume, the fraction of $\left|\vec{r}_{i j}\right|$, step function depending on the particle position, diagonal elements of the pressure tensor $P_{i j}$ with $P_{\mathrm{T}}=P_{x x}=P_{y y}$ and $P_{\mathrm{N}}=P_{z z}$, and $A_{0}$ is the value of Figure S2 (a) when the $\Sigma_{\text {mec }}=0$. The $z$ coordinate is perpendicular to the bilayers and $l_{\mathrm{z}}$ is the 
size along the $z$-direction of box. Then it can obtain $\eta=0.96, k_{r}=4.33$ in our simulation. Figure $\mathbf{S 3}$ exhibits the conversion processes from the $f^{e x}$ to the $\bar{v}$ and $\dot{\gamma}^{*} \cdot f^{\mathrm{ex}}=\left|\vec{f}^{\mathrm{ex}}\right|$.

This part is a supplementary explanation about the asphericity parameter. A tensor $\stackrel{T}{T}$ is constructed to characterize a configuration with $N$ unit masses, and its components can be expressed as $T_{i j}=\frac{1}{N} \sum_{l=1}^{N}\left(X_{i l}-\bar{X}_{i}\right)\left(X_{j l}-\bar{X}_{j}\right)(i, j=1,2, \ldots, \mathrm{d}) . X_{i l}$ is the $i$ th Cartesian component of the position vector of the $l$ th mass, and $\bar{X}_{i}=\frac{1}{N} \sum_{l=1}^{N} X_{i l}$ is the average of this component. For the three-dimensional case, the matrix has three eigenvalues, $\lambda_{1}, \lambda_{2}, \lambda_{3}$ the three principal radii of gyration squared.

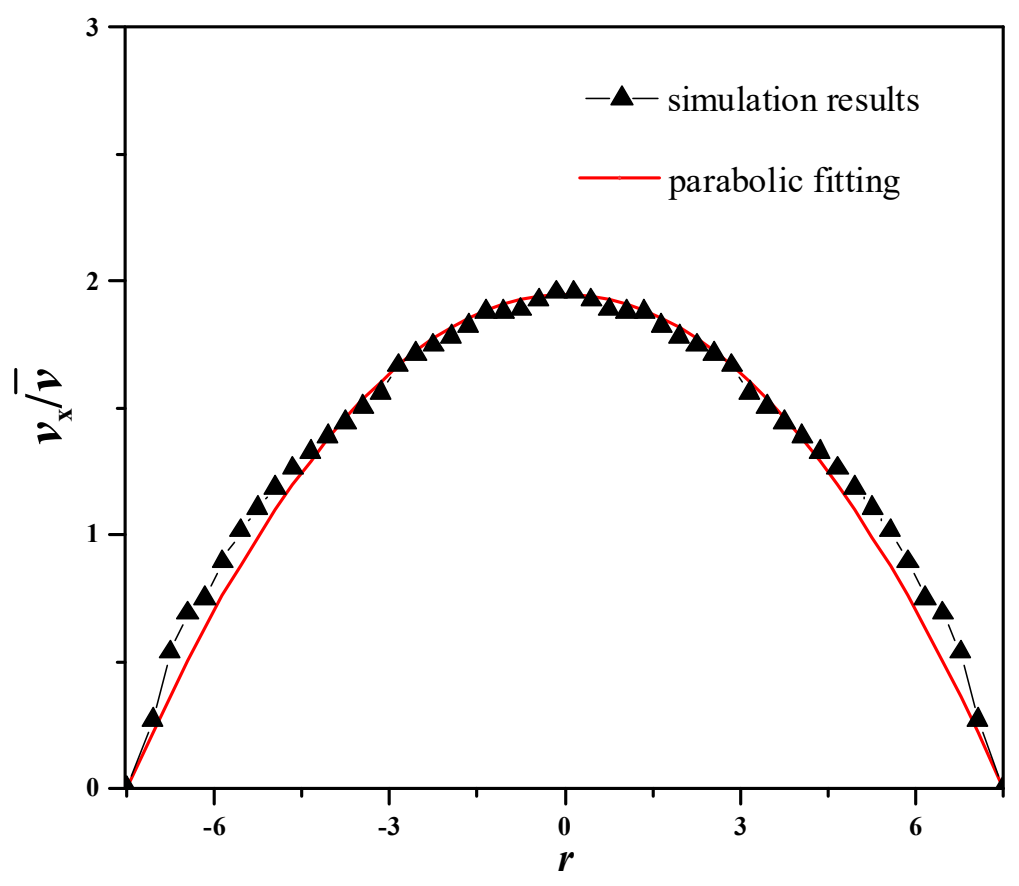

Figure S1. The velocity distribution of the solvent particles across the channel when a force $f^{\mathrm{ex}}=$ 24 is added to all the solvent particles under confinement degree $C_{\mathrm{d}}=0.8$. 
(a)

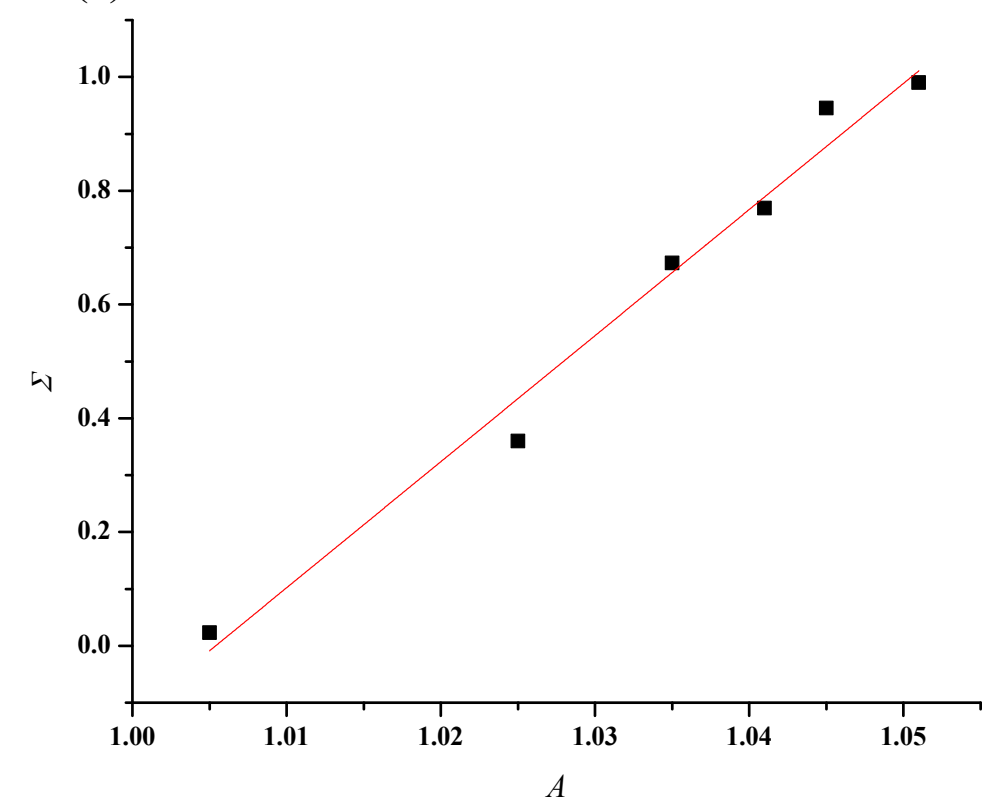

(b)

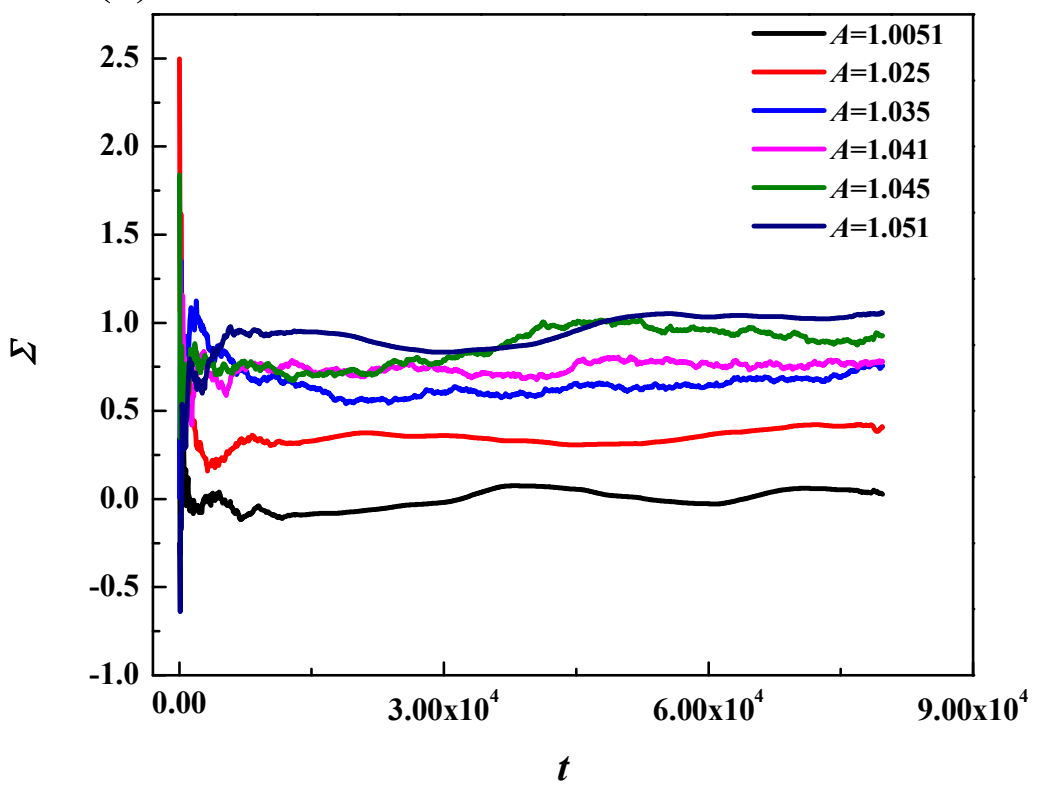


(c)

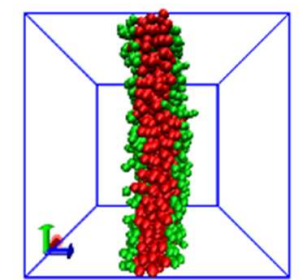

$A=1.005$

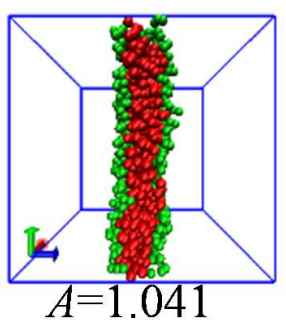

$A=1.041$

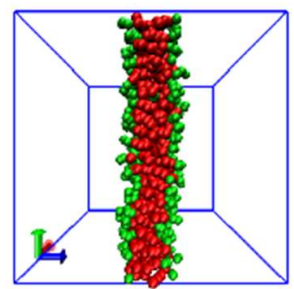

$A=1.025$

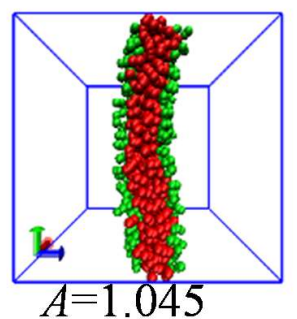

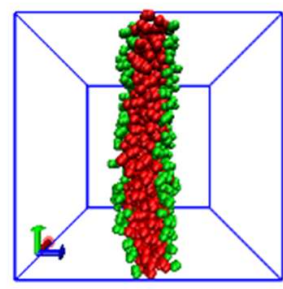

$A=1.035$

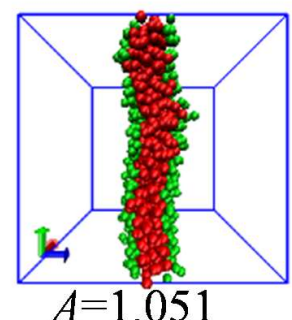

(d)

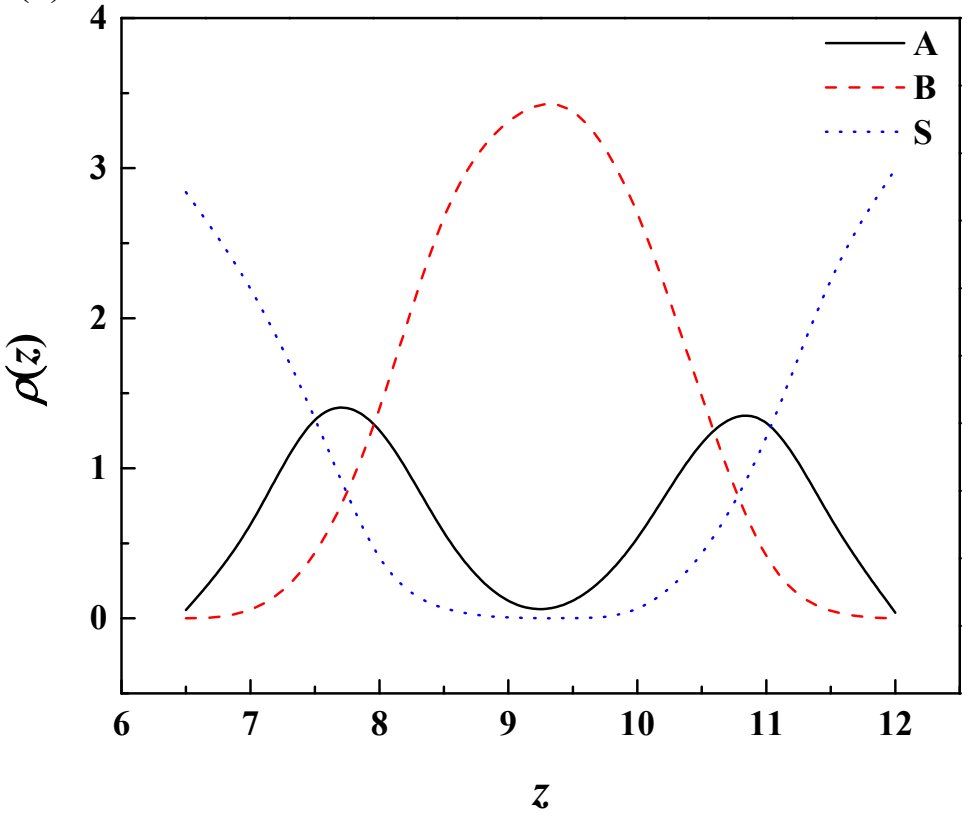

Figure S2. (a) the relationship of $A$ and $\Sigma$. (b) The mechanical membrane tensions $\Sigma$ change with time $t$ for different $A$. (c) The membrane of the $\mathrm{A}_{2} \mathrm{~B}_{4}$ vesicle used in text forms the stable bilayers 
snapshots, the value $A$ is the projected area per lipid. (d) The density profile $\rho$ of hydrophilic A, hydrophobic $\mathrm{B}$, solvents $\mathrm{S}$ beads of bilayer along the $z$ coordinate perpendicular to the bilayers. Note that normalizations have been chosen such that $L_{x} L_{y} \int_{0}^{L z} \rho_{\mathrm{D}}(z) \mathrm{d} z=N_{i}(i=\mathrm{A}, \mathrm{B}, \mathrm{S})$.

(a)

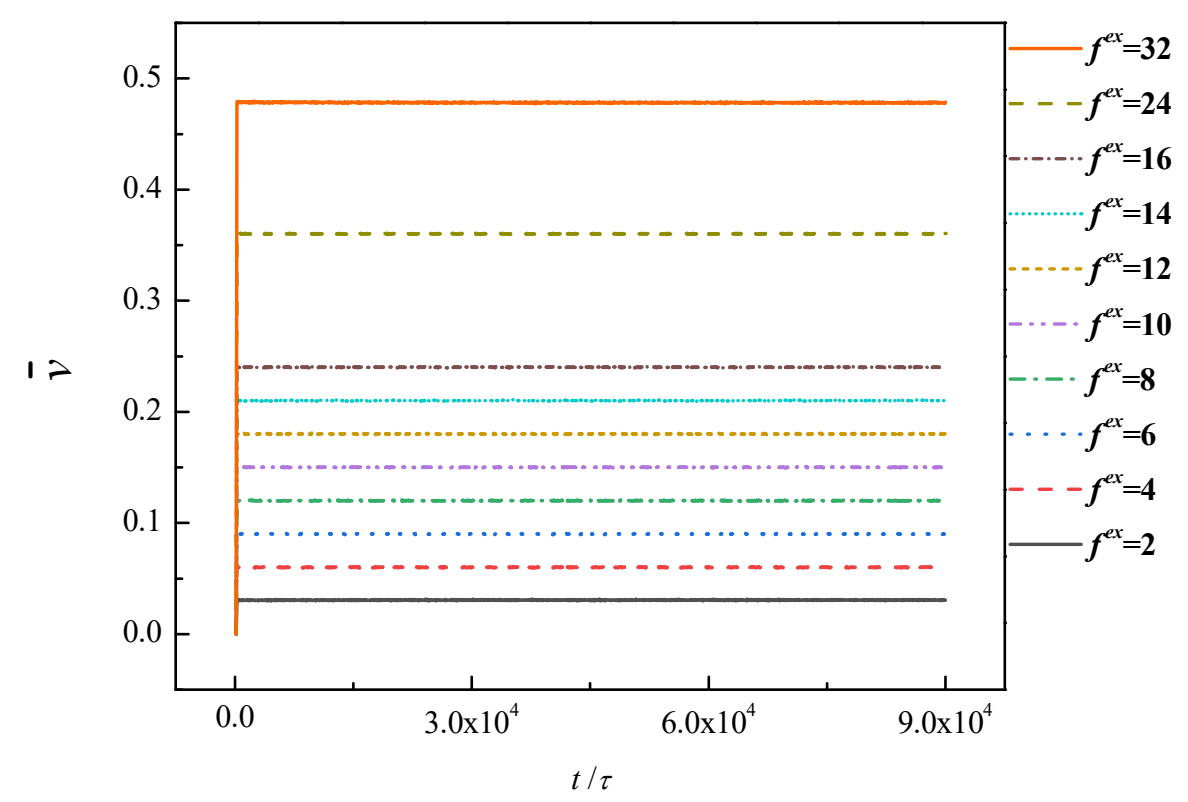


(b)

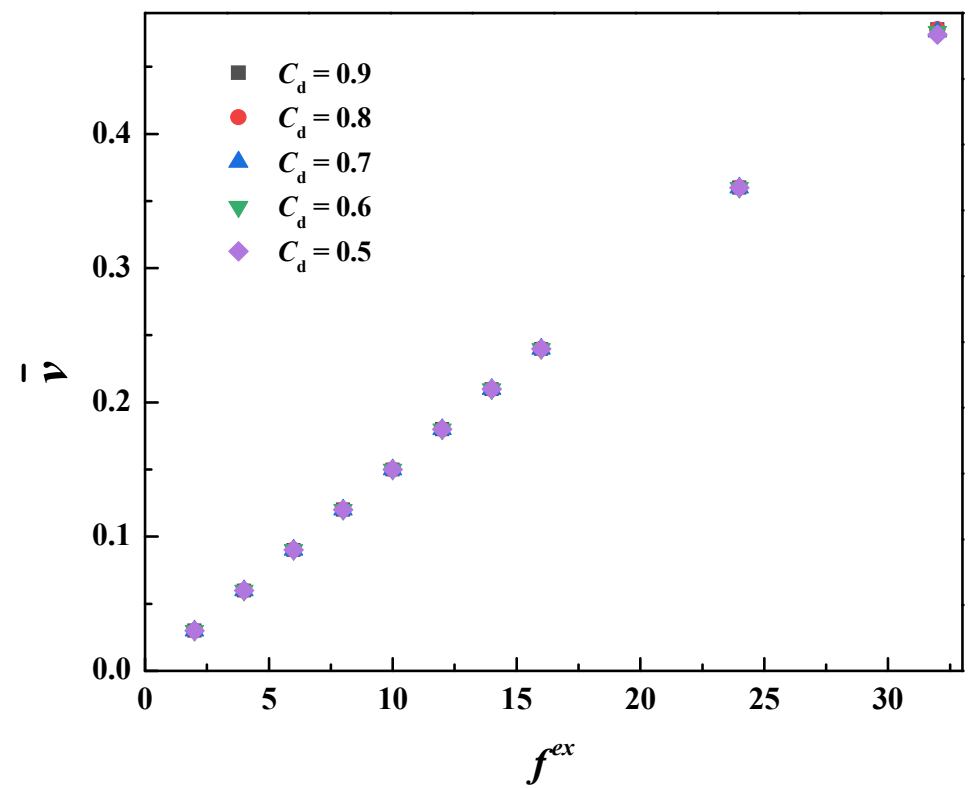

(c)

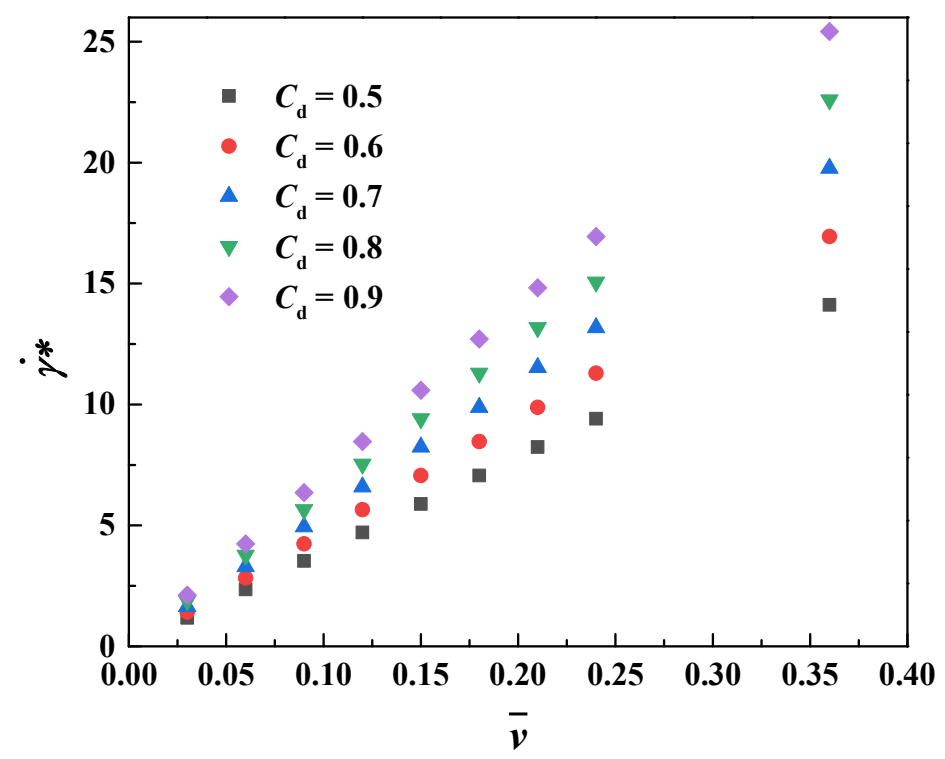

Figure S3. (a) Average fluidic flow velocity $\bar{v}$ varies with time for force different $f^{\text {ex }}$ applied on the solvents particles. (b) The linear relationship of $\dot{\gamma}^{*}$ and $f^{\mathrm{ex}}$. (c) The linear relationship of $\bar{v}$ and $\dot{\gamma}^{*}$. 


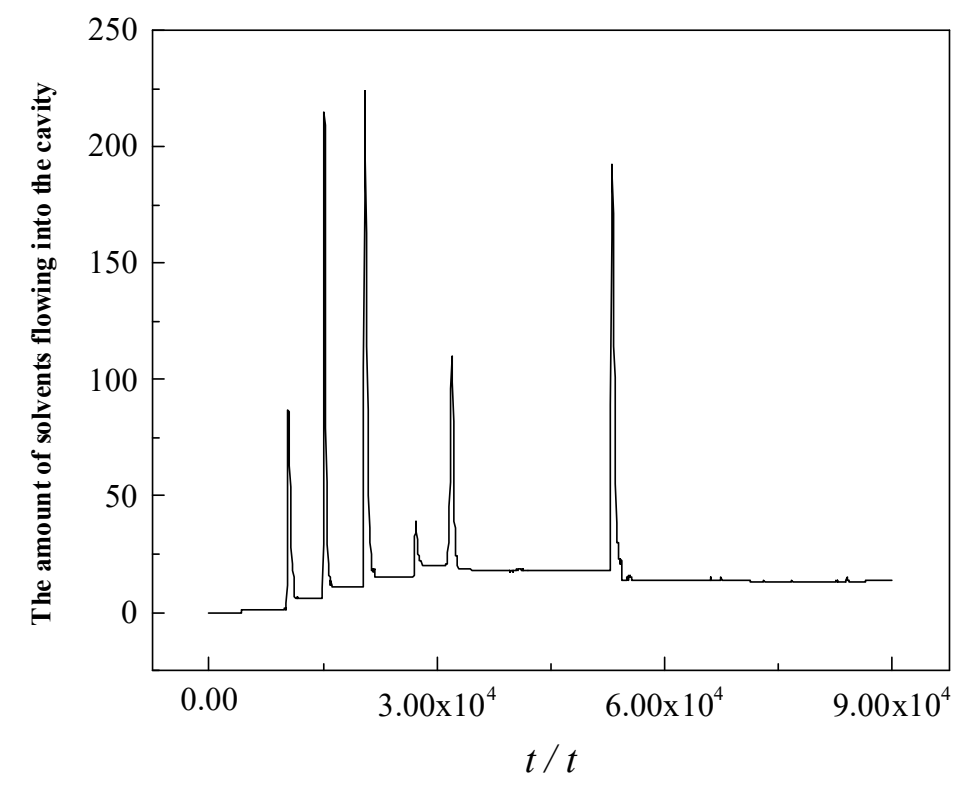

Figure S4. The number of solvents flowing into the cavity as a function of time with $\dot{\gamma}^{*}=10$ and $C_{\mathrm{d}}=0.5$.

\section{REFERENCES}

(1) Hoogerbrugge, P. J.; Koelman, J. M. V. A. Simulating Microscopic Hydrodynamic Phenomena with Dissipative Particle Dynamics. EPL 1992, 19, 155-160.

(2) Groot, R. D.; Warren, P. B. Dissipative Particle Dynamics: Bridging the Gap between Atomistic and Mesoscopic Simulation. J. Chem. Phys. 1997, 107, 4423-4435.

(3) Kroger, M.; Hess, S. Rheological Evidence for a Dynamical Crossover in Polymer Melts Via Nonequilibrium Molecular Dynamics. Phys. Rev. Lett. 2000, 85, 1128-1131.

(4) Heyes, D. M.; Smith, E. R.; Dini, D.; Zaki, T. A. The Equivalence between Volume Averaging and Method of Planes Definitions of the Pressure Tensor at a Plane. J. Chem. Phys. 2011, 135, 024512. 